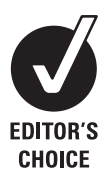

Li Ka Shing Institute of Health Sciences and Institute of Vascular Medicine, Division of Cardiology, Department of Medicine and Therapeutics, Prince of Wales Hospital, The Chinese University of Hong Kong, Hong Kong

Correspondence to: Professor Cheuk-Man Yu, Li Ka Shing Institute of Health Sciences, Institute of Vascular Medicine, Division of Cardiology, Department of Medicine and Therapeutics, Prince of Wales Hospital, The Chinese University of Hong Kong, Shatin, NT, Hong Kong; cmyu@cuhk.edu.hk

Accepted 1 July 2008 Published Online First 24 July 2008

\title{
Difference in long-term clinical outcome after cardiac resynchronisation therapy between ischaemic and non-ischaemic aetiologies of heart failure
}

\author{
O Zhang, J W-H Fung, J Y-S Chan, G Yip, Y-Y Lam, Y-J Liang, C-M Yu
}

\begin{abstract}
Objective: To examine the impact of heart failure (HF) aetiology on long-term outcome after cardiac resynchronisation therapy (CRT).
\end{abstract}

Design: Prospective cohort study.

Setting: University hospital.

Patients: 119 patients (44\% with ischaemic and 56\% non-ischaemic aetiology) who underwent CRT.

Interventions: Clinical follow-up for 39 (24) months.

Main outcome measures: Cardiovascular mortality, HF and cardiovascular hospitalisation were compared by Kaplan-Meier curves between the two groups, followed by Cox regression analysis for prognostic predictor(s).

Results: 41 (34\%) patients died, in whom cardiovascular causes were identified in 32 (27\%) patients. The ischaemic group had a higher cardiovascular mortality (log-rank $\chi^{2}=4.293, p=0.038$ ) and cardiovascular hospitalisation (log-rank $\chi^{2}=5.123, p=0.024$ ) when compared with the non-ischaemic group, though no difference was found in HF hospitalisation (log-rank $\left.\chi^{2}=0.019, p=0.892\right)$. At three months, left ventricular reverse remodelling occurred in $52 \%$ of the ischaemic group and $55 \%$ of the non-ischaemic group $\left(\chi^{2}=0.128\right.$, $p=0.720$ ). By Cox regression analysis, ischaemic aetiology and absence of reverse remodelling at three months were independent predictors of cardiovascular mortality $(H R=2.698, p=0.032 ; H R=3.541$, $\mathrm{p}=0.030$ ) and cardiovascular hospitalisation $(H R=1.905, p=0.015 ; H R=2.361, p=0.004)$. Furthermore, these two factors had an incremental value in predicting cardiovascular mortality when compared with either alone (left ventricular reverse remodelling, logrank $\chi^{2}=10.275$ vs 6.311, $p=0.05$; Ischaemic aetiology, log-rank $\chi^{2}=10.275$ vs 4.293, $p<0.05$ ).

Conclusion: Ischaemic aetiology of HF is an independent predictor of higher cardiovascular mortality and hospitalisation after CRT. This may implicate the progressive nature of coronary heart disease leading to a worse outcome despite similar short-term benefits of CRT.

Cardiac resynchronisation therapy (CRT) is an established treatment for patients with advanced heart failure (HF) who have electromechanical delay. Its beneficial effects on cardiac function, exercise capacity, quality of life, left ventricular (LV) reverse remodelling and long-term prognosis, including mortality and cardiovascular hospitalisation, have been confirmed in large clinical trials. ${ }^{1-7}$ In the MIRACLE study, a lesser degree of reduction of LV volumes at one-year follow-up was observed in ischaemic than non-ischaemic patients. ${ }^{89}$ However, it is not known whether there is any attributable difference in aetiology on long-term prognosis after CRT. In the CARE-HF study, when compared with ischaemic patients, non-ischaemic patients showed a greater extent of reduction in the estimated absolute risk for death or unplanned hospitalisation after receiving CRT, though aetiology of HF was not found to be an independent predictor for the outcome measure of CRT. ${ }^{10}$ On the other hand, early LV reverse remodelling occurring at 3-6 months after CRT detected by echocardiography has been shown to herald the improvement of long-term survival, apart from symptomatic improvement. ${ }^{3-5} 811$ Therefore, the main objective of the present study was to compare the long-term clinical outcome in patients with ischaemic and non-ischaemic aetiologies of HF after CRT. This will be tested in the multivariate model to examine if HF aetiology is an independent determinant of prognosis on top of short-term LV reverse remodelling.

\section{METHODS}

\section{Patients}

This study prospectively enrolled 119 patients with advanced HF (mean age 65 (SD 13) years, 75\% men) who underwent CRT and were followed up for at least three months. Four patients died (one refractory $\mathrm{HF}$, two sudden cardiac death (SCD), and one pneumonia) before the three-month follow-up, and were excluded from analysis as there was no echocardiographic follow-up data. The inclusion criteria for CRT included refractory HF despite optimal medical therapy, LV systolic dysfunction with ejection fraction $<40 \%$, and ORS duration $>120 \mathrm{~ms}$. Coronary heart disease was confirmed in 52 patients (44\%), who had past history of myocardial infarction and/or positive findings on coronary angiogram or underwent percutaneous coronary intervention. For the other 67 patients (56\%), non-ischaemic aetiology was confirmed by the normal coronary angiography. CRT devices were implanted as previously described. ${ }^{312}$ Only five patients in the ischaemic group and four patients in the non-ischaemic group had biventricular defibrillator devices, while all the others received biventricular pacemakers. Echocardiographic study with tissue Doppler imaging (TDI) and clinical assessment were performed serially before device implantation and at three months after CRT. Patients who had a decrease in LV end-systolic volume (LVESV) of $\geqslant 15 \%$ were defined as responders of LV reverse remodelling, and the others were classified as non-responders. The study protocol was approved by the ethics 
Table 1 Baseline clinical and echocardiographic characteristics in heart failure patients secondary to ischaemic and non-ischaemic aetiologies

\begin{tabular}{|c|c|c|c|}
\hline Parameters & Ischaemic $(n=52)$ & Non-ischaemic $(n=67)$ & p Value \\
\hline Age (years) & $65(12)$ & $64(13)$ & 0.699 \\
\hline \multicolumn{4}{|l|}{ Gender, $\%$ of patients } \\
\hline Male & 75 & 73 & $\chi^{2}=0.002$ \\
\hline Female & 25 & 27 & $p=0.962$ \\
\hline \multicolumn{4}{|l|}{ NYHA class, $\%$ of patients } \\
\hline III & 87 & 85 & $\chi^{2}=0.003$ \\
\hline IV & 13 & 15 & $p=0.950$ \\
\hline Quality of life score & $30(20)$ & $31(22)$ & 0.830 \\
\hline 6-Minute hall-walk distance (metres) & $306(113)$ & $338(107)$ & 0.135 \\
\hline \multicolumn{4}{|l|}{ Heart rhythm, $\%$ of patients } \\
\hline Sinus & 90 & 88 & $\chi^{2}=0.180$ \\
\hline Atrial fibrillation & 10 & 12 & $p=0.671$ \\
\hline LVEDV $\left(\mathrm{cm}^{3}\right)$ & $181(76)$ & $183(66)$ & 0.894 \\
\hline LVESV $\left(\mathrm{cm}^{3}\right)$ & $134(65)$ & $138(62)$ & 0.726 \\
\hline LV ejection fraction (\%) & $27.2(6.8)$ & $26.4(9.2)$ & 0.604 \\
\hline QRS duration (ms) & $131(31)$ & $137(37)$ & 0.354 \\
\hline Ts-SD (ms) & $35(12)$ & 39 (14) & 0.142 \\
\hline
\end{tabular}

LV, left ventricular; LVEDV, left ventricular end-diastolic volume; LVESV, left ventricular end-systolic volume; NYHA, New York Heart Association; Ts-SD, standard deviation of the time to peak myocardial systolic velocity among the 12 left ventricular segments.

committee of the university and written informed consent was obtained from all patients.

\section{Echocardiography}

Echocardiography with TDI was performed (Vivid 7, VingmedGeneral Electric, Horten, Norway) at baseline and three months after CRT. The atrioventricular interval was optimised by Ritter's method at day 1 after implantation to reach maximal transmitral diastolic filling. ${ }^{13}$ The LV volumes and ejection fraction were assessed by biplane Simpson's equation using the apical four-chamber and two-chamber views where the length of the ventricular image was maximised. At least three consecutive beats of sinus rhythm and 5-7 beats of atrial fibrillation were measured and the mean was calculated. LV sphericity indices, myocardial performance index and mitral regurgitation were measured as previously described. ${ }^{3} 14$ Furthermore, in the ischaemic group, wall motion score of individual LV segment was assessed ( $1=$ normal; $2=$ hypokinetic; $3=$ akinetic; $4=$ dyskinetic; $5=$ aneurysm) and hence wall motion score index of the 16 segments was calculated as previously described. ${ }^{15}$

Two-dimensional colour TDI was performed in the apical views (apical four-chamber, two-chamber and three-chamber views) and myocardial velocity curves were reconstituted (EchoPac PC, version 6.1.0, Vingmed-General Electric, Horten, Norway). The time to the peak systolic velocity at ejection phase was measured in each segment and systolic dyssynchrony was defined by calculating the dyssynchrony index (Ts-SD)that is, the standard deviation of time to the peak systolic velocity among the $12 \mathrm{LV}$ segments. ${ }^{3} 16-18$

Assessment of cardiovascular events during long-term follow-up All patients were followed up regularly (every two to three months) in the HF clinic, with clinical assessment, ECG recording and device interrogation to ensure biventricular pacing was maintained. The occurrence of cardiovascular events was adjudicated by two cardiologists blinded to the echocardiographic findings. Only those events happening after the threemonth follow-up were included in the analyses, in order to investigate the impact of short-term changes in cardiac function or clinical status on long-term prognosis. The cause of death was ascertained by reviewing the clinical record and investigation results, report of the close relatives and postmortem findings. Among cardiovascular hospitalisation and/or death, HF was defined according to clinical symptoms (limitation of activity, fatigue, and dyspnoea or orthopnoea), physical signs (oedema, elevated jugular venous pressure, rales or third heart sound with gallop) or radiological evidence of pulmonary congestion. ${ }^{19}$ The diagnosis of acute coronary syndrome was based on the current guidelines, including the presence of typical chest pain or discomfort, and elevation of cardiac enzymes such as creatine kinase- $\mathrm{MB}$ or troponin $\mathrm{T}$ and compatible ECG changes. In addition, cardiac arrhythmia, SCD and cerebrovascular accident were also included in cardiovascular causes of hospitalisation and/or death.

\section{Statistical analysis}

Results are presented as mean (SD). The paired or unpaired $t$ test was used when appropriate in the comparison of continuous variables between baseline and three months, or between the ischaemic and non-ischaemic groups. The Wilcoxon or Mann-Whitney test was adopted for the comparison of ordinal parameters accordingly. Comparison of proportions was performed using Pearson $\chi^{2}$ analysis. Multivariate logistic regression was used to find the independent predictor(s) for short-term LV reverse remodelling. Differences in long-term event-free survival between the ischaemic and non-ischaemic groups were compared by Kaplan-Meier survival curves where the log-rank $\chi^{2}$ values were presented. Cox regression multivariable survival analysis was used to evaluate potential predictor(s) for cardiovascular mortality. A p value $<0.05$ was considered statistically significant.

\section{RESULTS}

The baseline demographic, clinical and echocardiographic parameters are shown in table 1 . These parameters were all comparable between ischaemic and non-ischaemic groups without statistical differences. In addition, the wall motion score index was 2.0 (0.3) (range 1.4-2.8) in the ischaemic patients. On the first day after CRT, the optimised 
Table 2 Comparison of reverse remodelling and changes in other echocardiographic and clinical parameters at three months after cardiac resynchronisation therapy between ischaemic and non-ischaemic patients

\begin{tabular}{|c|c|c|c|c|c|c|c|}
\hline \multirow[b]{2}{*}{ Parameters } & \multicolumn{2}{|c|}{ All patients $(n=119)$} & \multicolumn{2}{|c|}{ Ischaemic $(n=52)$} & \multicolumn{2}{|c|}{ Non-ischaemic $(n=67)$} & \multirow{2}{*}{$\begin{array}{l}\text { Ischaemic vs } \\
\text { non- } \\
\text { ischaemic } \\
\text { p Value } \\
\end{array}$} \\
\hline & & $\mathrm{p}$ Value & & $\mathrm{p}$ Value & & p Value & \\
\hline$\Delta$ LVESV $\left(\mathrm{cm}^{3}\right)$ & $-26(30)$ & $<0.001$ & $-21(26)$ & $<0.001$ & $-31(33)$ & $<0.001$ & 0.082 \\
\hline$\Delta \mathrm{LV}$ ejection fraction (\%) & $7.4(6.9)$ & $<0.001$ & $5.9(6.0)$ & $<0.001$ & $8.6(7.4)$ & $<0.001$ & 0.032 \\
\hline$\Delta$ Sphericity index, end-diastole & $0.09(0.23)$ & $<0.001$ & $0.06(0.22)$ & 0.060 & $0.11(0.23)$ & 0.001 & 0.288 \\
\hline$\Delta \mathrm{MPI}$ & $-0.15(0.26)$ & $<0.001$ & $-0.12(0.29)$ & 0.016 & $-0.16(0.24)$ & $<0.001$ & 0.348 \\
\hline$\Delta \mathrm{LV}$ filling time (ms) & $59(147)$ & $<0.001$ & $38(125)$ & 0.057 & $76(161)$ & 0.001 & 0.204 \\
\hline$\Delta$ Quality of life score & $-9(22)$ & $<0.001$ & $-7(18)$ & 0.015 & $-11(24)$ & $<0.001$ & 0.267 \\
\hline$\Delta 6$-minute hall-walk distance (metres) & $32(81)$ & $<0.001$ & $22(93)$ & 0.141 & $40(70)$ & $<0.001$ & 0.256 \\
\hline \multicolumn{8}{|l|}{$\triangle$ NYHA class, $\%$ of patients } \\
\hline Improvement of two classes & 10 & & 8 & & 12 & & \\
\hline
\end{tabular}

$\Delta$, changes between three-month follow-up and baseline; LV, left ventricular; LVEDV, left ventricular end-diastolic volume; LVESV, left ventricular end-systolic volume; MPI: myocardial performance index; NYHA, New York Heart Association.

atrioventricular interval was 97 (26) ms, which was not different between ischaemic and non-ischaemic groups (95 (29) vs 98 (23) ms, p=0.559).

\section{Short-term reverse remodelling and its relation with systolic dyssynchrony and ischaemic or non-ischaemic aetiology}

At three months after CRT, LV reverse remodelling was achieved with a significant improvement in LV function and geometry, as well as clinical status (all p<0.001) (table 2). Sixtyfour patients $(54 \%)$ were found to be the volumetric responders, and the prevalence in the ischaemic group and non-ischaemic group was $52 \%$ and $55 \%$, respectively $\left(\chi^{2}=0.128, p=0.720\right)$. In the non-ischaemic group, all the measured clinical and echocardiographic parameters were observed to be improved. Similarly, favourable improvement was evident in most of the

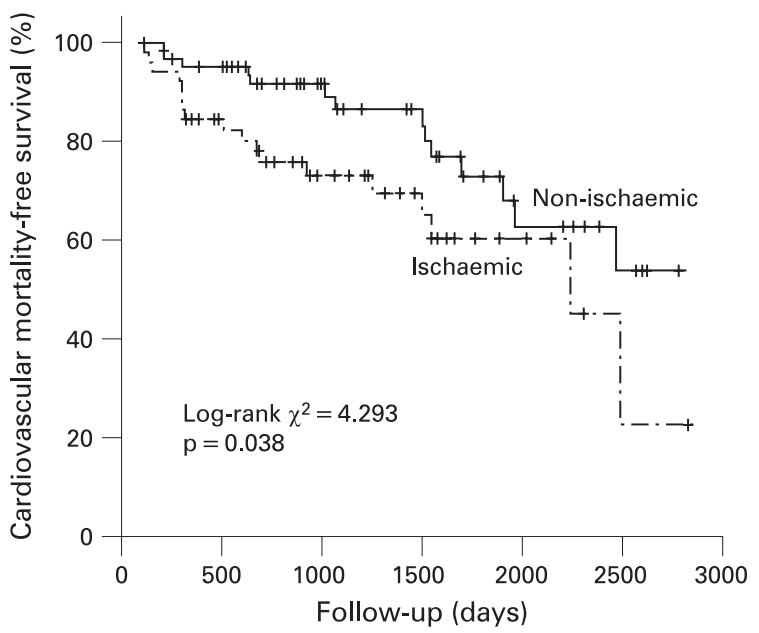

Number of patients at risk

$\begin{array}{lllllll}\text { Non-ischaemic } & 67 & 59 & 36 & 27 & 12 & 6 \\ \text { Ischaemic } & 52 & 39 & 24 & 14 & 6 & 1\end{array}$

Figure 1 Kaplan-Meier survival analysis showing a higher cardiovascular mortality in the ischaemic than non-ischaemic group after cardiac resynchronisation therapy. parameters in the ischaemic group, with the exception of enddiastolic sphericity index, LV filling time and 6-minute hallwalk distance. Moreover, in the ischaemic group, the wall motion score index was similar between the volumetric responders $(n=27)$ and non-responders $(n=25)(2.0(0.3)$ vs $2.1(0.3), p=0.548)$. When the extent of improvement was compared between the two aetiological groups, LVESV had a trend of greater reduction in the non-ischaemic group $(p=0.082)$, which gave rise to a greater increase in LV ejection fraction than in the ischaemic group $(p=0.032)$ (table 2$)$.

In the multivariate logistic regression analysis model for the prediction of short-term LV reverse remodelling response, severity of systolic dyssynchrony at baseline as measured by Ts-SD was the only independent covariate $(R R=1.191,95 \% \mathrm{CI}$ : 1.119 to $1.267 ; \mathrm{p}<0.001)$. Other factors including ischaemic versus non-ischaemic aetiology of HF, LV ejection fraction, ORS duration, age, gender and other clinical parameters were not significant. While $\mathrm{Ts}-\mathrm{SD} \geqslant 33 \mathrm{~ms}$ was adopted to define a significant dyssynchrony, ${ }^{16}{ }^{20}$ it was observed in $60 \%$ of the ischaemic group and $64 \%$ of the non-ischaemic group $\left(\chi^{2}=0.256, p=0.611\right)$.

\section{Clinical outcome of patients during long-term follow-up}

The mean duration of follow-up was 39 (24) months (range 392 months). One hundred and three patients (87\%) were followed up for more than one year, while ischaemic and nonischaemic patients had a comparable period of follow-up (36 (22) vs 43 (25) months, $p=0.128)$. Forty-one patients $(34 \%)$ died, in whom cardiovascular causes were identified in $32(27 \%)$, including HF in 12, SCD in 12, acute coronary syndrome in two, cardiogenic shock in one, ventricular fibrillation in two and cerebrovascular accident in three patients. The number of deaths was $22(42 \%)$ in the ischaemic group, with cardiovascular deaths in 18 (35\%) patients. These figures were $19(28 \%)$ and $14(21 \%)$, respectively, in the non-ischaemic group. In the ischaemic group the cardiovascular causes of death was HF in five, SCD in eight, acute coronary syndrome in two, ventricular fibrillation in one and cerebrovascular accident in two patients, and the corresponding figures were seven, four, 0 , one, one in the non-ischaemic group, plus cardiogenic shock in one patient. 


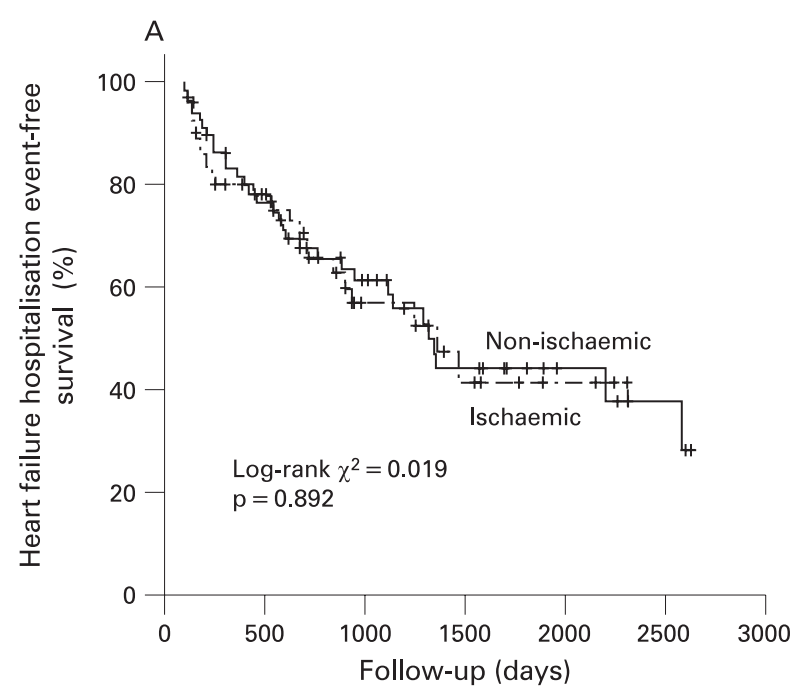

Number of patients at risk

$\begin{array}{lllllll}\text { Non-ischaemic } & 67 & 59 & 36 & 27 & 12 & 6 \\ \text { Ischaemic } & 52 & 39 & 24 & 14 & 6 & 1\end{array}$

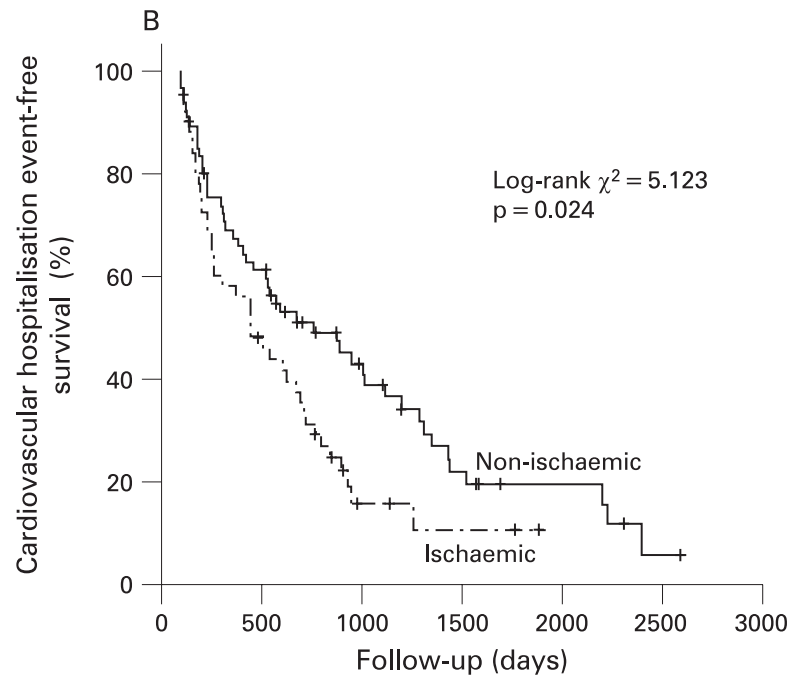

Number of patients at risk

$\begin{array}{lllllll}\text { Non-ischaemic } & 67 & 59 & 36 & 27 & 12 & 6 \\ \text { Ischaemic } & 52 & 39 & 24 & 14 & 6 & 1\end{array}$

Figure 2 Kaplan-Meier analysis for (A) heart failure (HF) hospitalisation event-free survival and (B) cardiovascular hospitalisation event-free survival that compared between the ischaemic and non-ischaemic groups. The ischaemic group had a lower cardiovascular hospitalisation event-free survival while the HF hospitalisation event-free survival was comparable between the two groups.
In the ischaemic group, 18 patients who had a cardiovascular death showed a trend of higher wall motion score index than those survived $(2.1(0.3)$ vs $2.0(0.3), p=0.075)$, though it was not statistically significant. In Kaplan-Meier survival analysis, it was demonstrated that patients with ischaemic aetiology had a significantly higher cardiovascular mortality (log-rank $\chi^{2}=4.293, p=0.038$ ) (fig 1 ), as well as all-cause mortality (log-rank $\chi^{2}=3.910, p=0.048$ ) during long-term follow-up than those with non-ischaemic aetiology.

During the long-term follow-up, 54 patients were hospitalised for fatal and non-fatal congestive $\mathrm{HF}$, which included 23 patients (44\%) in the ischaemic group and 31 patients $(46 \%)$ in the non-ischaemic group $(p=0.992)$. There was no difference in HF event-free survival between the two groups by KaplanMeier survival analysis (log-rank $\chi^{2}=0.019, p=0.892$ ) (fig 2A). Altogether there were 89 patients admitted for cardiovascular causes during long-term follow-up, with 41 patients (79\%) in the ischaemic group and 48 patients $(72 \%)$ in the non-ischaemic group ( $p=0.220$ ). However, the Kaplan-Meier survival analysis showed a significantly lower cardiovascular hospitalisation event-free survival in the ischaemic group when compared with the non-ischaemic group (log-rank $\left.\chi^{2}=5.123, p=0.024\right)$ (fig $2 B$ ).

Further investigation was performed by Kaplan-Meier survival analysis to examine the time course of changes in HF as well as cardiovascular hospitalisation event-free survival in the first, second and third-year follow-up in both ischaemic and nonischaemic groups. It was observed that there was no difference in both event rates in the first-year follow-up between ischaemic and non-ischaemic groups. However, a significantly lower cardiovascular hospitalisation event-free survival was observed in the ischaemic group in the second and third-year follow-up (table 3).

\section{Predictors of long-term event-free survival}

By using Cox multivariate regression analysis to predict longterm cardiovascular event-free survival, a group of covariates were selected into the model as shown in table 4 . These included clinical characteristics, aetiology of HF, as well as the three-month changes in echocardiographic parameters and clinical status. It was shown that ischaemic aetiology and the absence of short-term LV reverse remodelling at three months were independent predictors of cardiovascular mortality during long-term follow-up. Moreover, these two factors were found predictive of cardiovascular hospitalisation while the lack of LV reverse remodelling was the only predictor of HF hospitalisation (table 4).

Since ischaemic aetiology and the lack of LV reverse remodelling at three months appeared to be independent

Table 3 Comparison of the heart failure (HF) and cardiovascular hospitalisation event-free survival in ischaemic and non-ischaemic patients after cardiac resynchronisation therapy at the end of first, second and third-year follow-up

\begin{tabular}{|c|c|c|c|}
\hline $\begin{array}{l}\text { Hospitalisation event-free survival } \\
\text { (\% of patients) }\end{array}$ & $\begin{array}{l}\text { Ischaemic } \\
\text { (n= 52) }\end{array}$ & $\begin{array}{l}\text { Non-ischaemic } \\
(n=67)\end{array}$ & Ischaemic vs non-ischaemic \\
\hline \multicolumn{4}{|l|}{ First-year follow-up: } \\
\hline HF related & $74 \%$ & $80 \%$ & log-rank $\chi^{2}=1.053, p=0.305$ \\
\hline Cardiovascular related & $51 \%$ & $61 \%$ & log-rank $\chi^{2}=2.316, p=0.128$ \\
\hline \multicolumn{4}{|l|}{ Second-year follow-up: } \\
\hline HF related & $65 \%$ & $68 \%$ & log-rank $\chi^{2}=0.432, p=0.511$ \\
\hline Cardiovascular related & $27 \%$ & $49 \%$ & log-rank $\chi^{2}=6.150, p=0.013$ \\
\hline \multicolumn{4}{|l|}{ Third-year follow-up: } \\
\hline HF related & $54 \%$ & $67 \%$ & log-rank $\chi^{2}=2.729, p=0.099$ \\
\hline Cardiovascular related & $10 \%$ & $44 \%$ & log-rank $\chi^{2}=11.443, p=0.001$ \\
\hline
\end{tabular}


Table 4 Prediction of long-term cardiovascular events using multivariate Cox regression analysis

\begin{tabular}{|c|c|c|c|c|c|c|}
\hline \multirow[b]{2}{*}{ Parameters } & \multicolumn{2}{|l|}{ Cardiovascular mortality } & \multicolumn{2}{|l|}{ HF hospitalisation } & \multicolumn{2}{|c|}{ Cardiovascular hospitalisation } \\
\hline & HR $(95 \%$ Cl) & p Value & HR $(95 \%$ Cl) & p Value & HR $(95 \% \mathrm{Cl})$ & p Value \\
\hline Age & $1.029(0.995$ to 1.064$)$ & 0.099 & $1.022(0.998$ to 1.046$)$ & 0.079 & 1.012 (0.994 to 1.032$)$ & 0.196 \\
\hline Female gender & $1.498(0.589$ to 3.805$)$ & 0.396 & 1.565 (0.778 to 3.147$)$ & 0.209 & 1.242 (0.710 to 2.175$)$ & 0.448 \\
\hline Sinus rhythm & 0.409 (0.146 to 1.149$)$ & 0.090 & $1.637(0.568$ to 4.715$)$ & 0.361 & 0.932 (0.452 to 1.919$)$ & 0.848 \\
\hline Ischaemic aetiology & 2.698 (1.092 to 6.667$)$ & 0.032 & $1.216(0.638$ to 2.318$)$ & 0.552 & 1.905 (1.135 to 3.196$)$ & 0.015 \\
\hline$\Delta$ LVESV $<15 \%$ & 3.541 (1.131 to 11.079$)$ & 0.030 & 2.385 (1.106 to 5.142$)$ & 0.027 & 2.361 (1.314 to 4.243$)$ & 0.004 \\
\hline$\Delta \mathrm{LV}$ ejection fraction $<5 \%$ & 1.155 (0.436 to 3.059$)$ & 0.772 & 1.014 (0.490 to 2.098$)$ & 0.969 & $0.750(0.421$ to 1.334$)$ & 0.327 \\
\hline$\Delta$ NYHA class & $1.084(0.510$ to 2.300$)$ & 0.834 & $1.013(0.585$ to 1.754$)$ & 0.963 & $1.141(0.744$ to 1.750$)$ & 0.546 \\
\hline
\end{tabular}

$\mathrm{Cl}$, confidence interval; HF, heart failure; HR, hazard ratio; LV, left ventricular; LVESV, left ventricular end-systolic volume; MPI: myocardial performance index; NYHA, New York Heart Association.

long-term prognosticators of CRT, patients were divided further into four groups with respect to the status of short-term LV reverse remodelling and aetiology of HF. This included nonischaemic patients with reverse remodelling (group 1, $n=37$ ), non-ischaemic patients without reverse remodelling (group 2, $\mathrm{n}=30$ ), ischaemic patients with reverse remodelling (group 3 , $\mathrm{n}=27$ ) and ischaemic patients without reverse remodelling (group 4, $\mathrm{n}=25$ ). The cardiovascular mortality compared by Kaplan-Meier survival analysis was shown to be different among these groups (log-rank $\chi^{2}=10.275, p=0.016$ ) (fig 3). Further analysis revealed that the non-ischaemic patients with reverse remodelling had the lowest cardiovascular mortality (group 1 vs group 2: log-rank $\chi^{2}=4.993, p=0.025$; group 1 vs group 3: log-rank $\chi^{2}=4.513, p=0.034$; group 1 vs group 4: logrank $\left.\chi^{2}=10.332, p=0.001\right)$. The differences among the other three groups were not statistically significant.

Furthermore, the absence of LV reverse remodelling and ischaemic aetiology have incremental prognostic values. Combining both parameters became significantly superior in predicting cardiovascular mortality when compared with either single factor (fig 4).

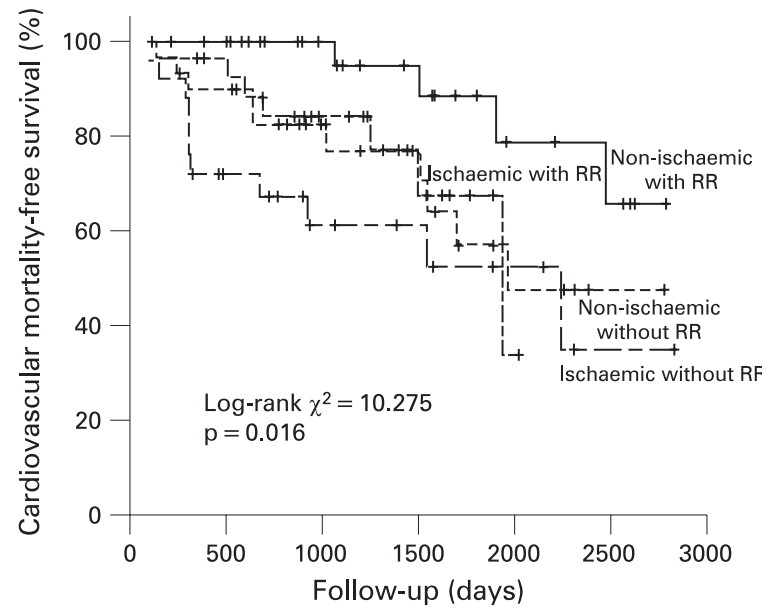

Number of patients at risk

$\begin{array}{lllllll}\text { Non-ischaemic } & 67 & 59 & 36 & 27 & 12 & 6 \\ \text { Ischaemic } & 52 & 39 & 24 & 14 & 6 & 1\end{array}$

Figure 3 Comparison of cardiovascular mortality by Kaplan-Meier survival analysis among the four groups of patients-non-ischaemic patients with left ventricular reverse remodelling $(R R)(n=37)$, nonischaemic patients without $R R(n=30)$, ischaemic patients with $R R$ $(n=27)$ and ischaemic patients without $R R(n=25)$. The non-ischaemic patients with RR had the best cardiovascular death event-free survival.

\section{DISCUSSION}

This study illustrated the impact of HF aetiology on long-term prognosis after CRT. During the follow-up with a mean duration of over three years, ischaemic patients were associated with a significantly higher cardiovascular event rate than non-ischaemic patients. Interestingly, the difference in cardiovascular hospitalisation became more apparent when patients were followed up for longer periods, in particular after two years. Furthermore, the prognostic importance of HF aetiology appeared to be independent of short-term LV reverse remodelling.

\section{Ischaemic aetiology is associated with worse long-term clinical outcome after CRT}

In our study, ischaemic patients were found to have higher cardiovascular mortality and lower cardiovascular event-free survival during the long-term follow-up after CRT. It is intriguing to note that cardiovascular hospitalisation event-free survival was not different in the first year between the two aetiological groups, though the survival curve continued to diverse over time and therefore in the second and third year, the event-free survival became significantly lower in the ischaemic group. From our observation, we postulate that CRT improves the uncoordinated contraction in both ischaemic and non-ischaemic aetiologies of

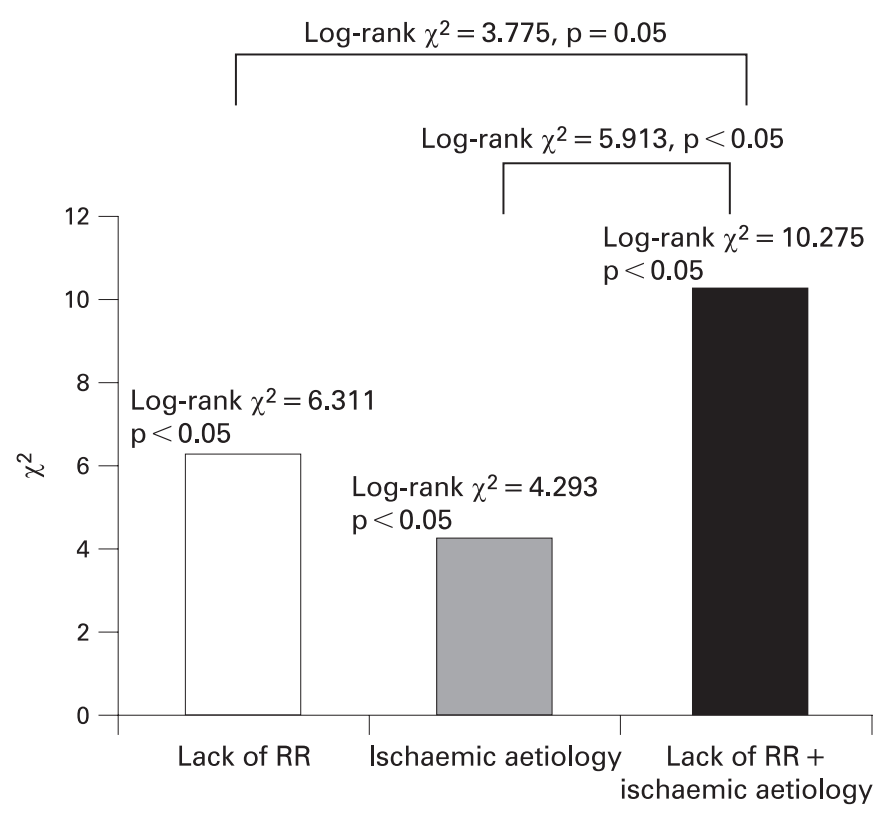

Figure 4 Incremental predictive value of combining the lack of early left ventricular reverse remodelling (RR) and ischaemic aetiology for cardiovascular mortality on top of the individual predictor. 
HF when significant systolic dyssynchrony is present. This leads to an early improvement of haemodynamics, increase in systolic function and favourable LV reverse remodelling which heralds the improvement of long-term prognosis leading to a lower mortality and a lower cardiovascular hospitalisation event rate. On the other hand, CRT is not a treatment for myocardial ischaemia itself. As a result, when the underlying coronary heart disease progresses, it might lead to the subsequent development of fatal and non-fatal cardiovascular events, which include acute coronary syndrome, arrhythmia and so on. This effect will only be revealed when patients were followed up for a long period of time.

In the current study, although the ischaemic group showed a trend of lesser degree of improvement in LVESV at three months, the volumetric responder rate was similar between the two groups by using a reduction of LVESV $\geqslant 15 \%$. Therefore, the impact of heart failure aetiology on long-term prognosis may operate independently of whether short-term LV reverse remodelling has occurred or not. In fact, the InSync/InSync-ICD Italian Registries observed a significantly higher all-cause mortality in ischaemic patients during three-year follow-up even when CRT benefits on clinical symptoms and echocardiographic parameters persisted. ${ }^{21}$ In the MIRACLE study, LV reverse remodelling occurred at six months and persisted in both ischaemic and non-ischaemic cardiomyopathy at 12 months, though this occurred to a lesser degree in ischaemic patients. ${ }^{9}$ It is not clear, however, from the study whether such inferiority in mediumterm cardiac response would result in a worse long-term prognosis in ischaemic aetiology of HF. The recent analysis of CARE-HF failed to identify ischaemic aetiology as an independent predictor for worse prognosis after CRT.$^{10}$ Nevertheless, the study found a higher estimated absolute risk in ischaemic than in non-ischaemic patients (0.63 vs 0.41$)$ for death from any cause or unplanned hospitalisation for cardiovascular events. ${ }^{10}$

\section{HF aetiology and short-term LV reverse remodelling after CRT}

Although previous studies have demonstrated short-term LV reverse remodelling response after CRT, ${ }^{7-9}{ }^{11}$ the present study observed that there were no significant differences between ischaemic and non-ischaemic cardiomyopathy, in both the extent of changes of LV volume and the proportion of responders. Of note, there was also no difference in the severity of baseline dyssynchrony between ischaemic and non-ischaemic groups, as shown by the dyssynchrony index (Ts-SD), a finding consistent with our pervious observations. ${ }^{17}$

Difference in LV reverse remodelling response with respect to different aetiologies had been reported in previous studies. In the MIRACLE study, it has been suggested that non-ischaemic patients had a greater degree of reverse remodelling with a higher ejection fraction than ischaemic patients after CRT for 612 months. $^{89}$ Our current study also found that the gain in ejection fraction is greater in the non-ischaemic group. This is in contrast to the study by Molhoek et al who did not report any difference in ejection fraction at six-month follow-up..$^{22}$ On the other hand, previous studies did not provide insight into whether there was any difference in the severity of systolic dyssynchrony. As previous studies also demonstrated that baseline dyssynchrony was a major determinant of short-term reverse remodelling response, the observed difference in response between the two aetiologies in various studies could have been the result of the difference in severity of systolic dyssynchrony. ${ }^{17}$

Our current study also illustrated the independent and incremental values of HF aetiology and short-term LV reverse remodelling response on long-term prognosis after CRT.
Therefore, the best clinical outcome occurs in non-ischaemic patients who have also shown early LV reverse remodelling at three months after CRT.

Funding: This study was supported by a research grant from Li Ka Shing Institute of Health Sciences.

Competing interests: None.

Ethics approval: Obtained.

Patient consent: Written informed consent was obtained from all patients.

\section{REFERENCES}

1. Cazeau S, Leclercq C, Lavergne T, et al. Effects of multisite biventricular pacing in patients with heart failure and intraventricular conduction delay. $N$ Engl J Med 2001;344:873-80.

2. Abraham WT, Fisher WG, Smith AL, et al. Cardiac resynchronization in chronic heart failure. N Engl J Med 2002;346:1845-53.

3. Yu CM, Chau E, Sanderson JE, et al. Tissue Doppler echocardiographic evidence of reverse remodeling and improved synchronicity by simultaneously delaying regional contraction after biventricular pacing therapy in heart failure. Circulation 2002;105:438-45.

4. Bradley DJ, Bradley EA, Baughman KL, et al. Cardiac resynchronization and death from progressive heart failure: a meta-analysis of randomized controlled trials. JAMA 2003:289:730-40.

5. Cleland JG, Daubert JC, Erdmann E, et al. The effect of cardiac resynchronization on morbidity and mortality in heart failure. N Engl J Med 2005;352:1539-49.

6. Bristow MR, Saxon LA, Boehmer J, et al. Cardiac-resynchronization therapy with or without an implantable defibrillator in advanced chronic heart failure. $N$ Engl J Med 2004:350:2140-50.

7. Sutton MS, Keane MG. Reverse remodelling in heart failure with cardiac resynchronisation therapy. Heart 2007;93:167-71.

8. St John Sutton MG, Plappert T, Abraham WT, et al. Effect of cardiac resynchronization therapy on left ventricular size and function in chronic heart failure. Circulation 2003;107:1985-90.

9. Sutton MG, Plappert T, Hilpisch KE, et al. Sustained reverse left ventricular structural remodeling with cardiac resynchronization at one year is a function of etiology: quantitative Doppler echocardiographic evidence from the Multicenter InSync Randomized Clinical Evaluation (MIRACLE). Circulation 2006;113:266-72.

10. Richardson M, Freemantle N, Calvert MJ, et al. Predictors and treatment response with cardiac resynchronization therapy in patients with heart failure characterized by dyssynchrony: a pre-defined analysis from the CARE-HF trial. Eur Heart $J$ 2007;28:1827-34.

11. Yu CM, Bleeker GB, Fung JW, et al. Left ventricular reverse remodeling but not clinical improvement predicts long-term survival after cardiac resynchronization therapy. Circulation 2005;112:1580-6.

12. Daubert JC, Ritter $\mathrm{P}$, Le Breton $\mathrm{H}$, et al. Permanent left ventricular pacing with transvenous leads inserted into the coronary veins. Pacing Clin Electrophysiol 1998:21:239-45

13. Ritter $\mathbf{P}$, Padeletti L, Gillio-Meina $L$, et al. Determination of the optimal atrioventricular delay in DDD pacing. Comparison between echo and peak endocardial acceleration measurements. Europace 1999;1:126-30.

14. Naqvi TZ, Goel RK, Forrester JS, et al. Myocardial contractile reserve on dobutamine echocardiography predicts late spontaneous improvement in cardiac function in patients with recent onset idiopathic dilated cardiomyopathy. J Am Coll Cardiol 1999;34:1537-44.

15. Schiller NB, Shah PM, Crawford M, et al. Recommendations for quantitation of the left ventricle by two-dimensional echocardiography. American Society of Echocardiography Committee on Standards, Subcommittee on Quantitation of TwoDimensional Echocardiograms. J Am Soc Echocardiogr 1989;2:358-67.

16. Yu CM, Lin H, Zhang 0, et al. High prevalence of left ventricular systolic and diastolic asynchrony in patients with congestive heart failure and normal QRS duration. Heart 2003; 89:54-60.

17. Yu CM, Fung JW, Zhang 0 , et al. Tissue Doppler imaging is superior to strain rate imaging and postsystolic shortening on the prediction of reverse remodeling in both ischemic and nonischemic heart failure after cardiac resynchronization therapy. Circulation 2004;110:66-73.

18. Zhang 0, Fung JW, Auricchio A, et al. Differential change in left ventricular mass and regional wall thickness after cardiac resynchronization therapy for heart failure. Eur Heart J 2006;27:1423-30.

19. The effect of digoxin on mortality and morbidity in patients with heart failure. The Digitalis Investigation Group. N Engl J Med 1997;336:525-33.

20. Yu CM, Fung JWH, Lin $\mathrm{H}$, et al. Predictors of left ventricular reverse remodeling after cardiac resynchronization therapy for heart failure secondary to idiopathic dilated or ischemic cardiomyopathy. Am J Cardiol 2003;91:684-8.

21. Gasparini M, Lunati $\mathbf{M}$, Santini $M$, et al. Long-term survival in patients treated with cardiac resynchronization therapy: a 3-year follow-up study from the InSync/InSync ICD Italian Registry. Pacing Clin Electrophysiol 2006;29:S2-10.

22. Molhoek SG, Bax JJ, van Erven L, et al. Comparison of benefits from cardiac resynchronization therapy in patients with ischemic cardiomyopathy versus idiopathic dilated cardiomyopathy. Am J Cardiol 2004;93:860-3. 